\title{
Goos-Hänchen shifts in frustrated total internal reflection studied with wave packet propagation
}

\author{
Xi Chen ${ }^{1,2 *}$, Chun-Fang $\mathrm{Li}^{1,3}$, Rong-Rong $\mathrm{Wei}^{1}$, and Yan Zhang ${ }^{4}$ \\ 1 Department of Physics, Shanghai University, 200444 Shanghai, People's Republic of China \\ 2 Departamento de Química-Física, UPV-EHU, Apdo 644, 48080 Bilbao, Spain \\ 3 State Key Laboratory of Transient Optics and Photonics, \\ Xi'an Institute of Optics and Precision Mechanics of CAS, 710119 Xi'an, People's Republic of China and \\ 4 Department of Electronics and Information Engineering, \\ Shanghai University, 200072 Shanghai, People's Republic of China
}

(Dated: November 7, 2018)

\begin{abstract}
We have investigated that the Goos-Hänchen (GH) shifts in frustrated total internal reflection (FTIR) studied with wave packet propagation. In the first-order approximation of the transmission coefficient, the GH shift is exactly the expression given by stationary phase method, thus saturates an asymptotic constant in two different ways depending on the angle of incidence. Taking account into the second-order approximation, the GH shift always depends on the width of the air gap due to the modification of the beam width. It is further shown that the GH shift with second-order correction increases with decreasing the beam width at the small incidence angles, while for the large incidence angles it reveals a strong decrease with decreasing the beam width. These phenomena offers the better understanding of the tunneling delay time in FTIR.
\end{abstract}

PACS numbers: 42.25.Bs, 42.25.Gy, 73.40.Gk

It is well known that a light beam totally reflected from an interface between two dielectric media undergoes lateral shift from the position predicted by geometrical optics [1]. This phenomenon was referred to as Goos-Hänchen (GH) effect and was theoretically explained by Artmann's stationary phase method [3] and Renard's energy flux method [4]. Because of the potentials applications in integrated optics [2], optical waveguide switch [5], and optical sensors [6, 7], the GH shifts including other three non-specular effects such as angular deflection, focal shift and waist-width modification have been extensively investigated in partial reflection 8, 9, 10, 11, 12, 13], attenuated total reflection 14, 15], and frustrated total internal reflection (FTIR) [16, 17, 18, 19, 20].

From a somewhat different perspective, the optical tunneling phenomenon in FTIR have attracted much attention in the last two decades [21, 22, 23, 24, 25], because of the analogy between FITR and quantum tunneling. Theoretical 21, 22] and experimental [23, 24] investigations have demonstrated that the GH shifts in FTIR play an important role in the superluminal tunneling time and the well-known "Hartman effect" [26], which describes that the group delay for quantum particles tunneling though a potential barrier saturates to a constant for an opaque barrier. Recently, Martinez and Polatdemir [27] have studied the effect of width of the beam on the GH shift (which is proportional to tunneling time) to offer the complementary insights into the origin of "Hartman effect" in FTIR. In addition, Haibel et al. 19] once carried out a comprehensive study of

\footnotetext{
*Email address: xchen@shu.edu.cn
}

the GH shift in FTIR as a function of the polarization, beam width, and incidence angle in the microwave experiment, which challenges its theoretical descriptions. However, the current expressions of the GH shifts given by stationary phase method and energy flux method are independent of the beam width.

The main purpose of this Brief Report is to investigate that the GH shifts in FTIR by wave packet propagation. It is shown that the GH shift in the first-order approximation of the transmission coefficient is exactly the expression of the GH shift given by stationary phase method. The GH shift in this case approaches the saturation value in two different ways depending on the incidence angle. Taking account into the second-order approximation, the GH shift always depends on the width of air gap. It is further shown that the GH shift with the second-order correction become dependent strongly on the beam width. These phenomena offers the better understanding of the tunneling delay time in FTIR.

For simplicity, we consider TE polarized beam incident into the double-prism structure with the angular frequency $\omega$ and incidence angle $\theta_{0}$, as shown in Fig. 1, where $a$ is the width of the air gap. Denote by $\varepsilon, \mu$ and $n$, respectively, the permittivity, permeability and refractive index of the prism. For a well-collimated beam, the electric field of the incident beam can be expressed by

$$
\Psi_{i n}(x)=\frac{1}{\sqrt{2 \pi}} \int_{-\infty}^{\infty} A\left(k_{y}\right) \exp \left[i\left(k_{x} x+k_{y} y\right)\right] d k_{y},
$$

where $k_{x}=n k \cos \theta, k_{y}=n k \sin \theta, k=\omega / c, n=\sqrt{\epsilon \mu}, c$ is the speed of light in vacuum, $\theta$ is the incident angle of the plane wave component under consideration, and time dependence $\exp (-i \omega t)$ is implied and suppressed. For a Gaussian-shaped incident beam whose peak is assumed to be located at $x=0$, 


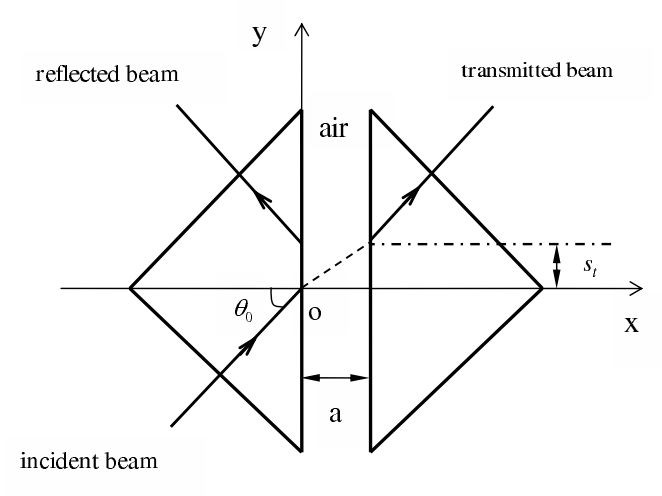

FIG. 1: Schematic diagram of the GH shift in FTIR, where the width of the air gap is $a, s_{t}$ represents the GH shift of the transmitted beam.

$$
\Psi_{i}(x=0, y)=\exp \left(-\frac{y^{2}}{2 w_{y}^{2}}\right) \exp \left(i k_{y 0} y\right),
$$

its angular spectral distribution is also a Gaussian function, $A\left(k_{y}\right)=w_{y} \exp \left[-\left(w_{y}^{2} / 2\right)\left(k_{y}-k_{y 0}\right)^{2}\right]$, around its central $k_{y 0}=k \sin \theta_{0}, w_{y}=w_{0} / \cos \theta_{0}, w_{0}$ is the width of the beam at waist. According to Maxwell equations and boundary conditions, the field of the transmitted beam is found to be

$$
\Psi_{t}(x)=\frac{1}{\sqrt{2 \pi}} \int_{-\infty}^{\infty} T A\left(k_{y}\right) \exp \left\{i\left[k_{x}(x-a)+k_{y} y\right]\right\} d k_{y},
$$

with the transmitted coefficient $T=\exp (i \phi) / f$ is given by

$$
f \exp (i \phi)=\cosh \kappa a+\frac{i}{2}\left(\frac{k_{x}}{\kappa}-\frac{\kappa}{k_{x}}\right) \sinh \kappa a,
$$

where $\kappa=\left(k_{y}^{2}-k^{2}\right)^{1 / 2}$.

Firstly, we look at the GH shift in the first-order approximation of the transmission coefficient. Expand the exponent in Taylor series at $k_{y 0}$, and retain up to the first-order term, then we will obtain

$$
T\left(k_{y}\right)=\exp \left[\ln T\left(k_{y}\right)\right] \approx T_{0} \exp \left[\frac{1}{T_{0}} \frac{d T}{d k_{y 0}}\left(k_{y}-k_{y 0}\right)\right]
$$

where $T_{0}=T\left(k_{y 0}\right)$, and $d / d k_{y 0}$ denotes the derivative with respect to $k_{y}$ evaluated at $k_{y}=k_{y 0}$. Introduce two real parameters $L_{t}^{\prime}$ and $L_{t}^{\prime \prime}$ defined as,

$$
L=L_{t}^{\prime}+i L_{t}^{\prime \prime}=\frac{i}{T_{0}} \frac{d T}{d k_{y 0}},
$$

then, in terms of the phase and magnitude of $T$, we will have

$$
L_{t}^{\prime}=-\frac{d \phi}{d k_{y 0}}
$$

and

$$
L_{t}^{\prime \prime}=\frac{d}{d k_{y 0}} \ln \left|T\left(k_{y}\right)\right|
$$

Substituting Eq. (5) into Eq. (3) and employing the paraxial approximation condition,

$$
k_{x} \approx k_{x 0}-\left(k_{y}-k_{y 0}\right) \tan \theta_{0}-\frac{\left(k_{y}-k_{y 0}\right)^{2}}{2 k \cos ^{2} \theta_{0}},
$$

we obtain the transmitted beam at $x=a$,

$$
\Psi_{t}(a, y) \approx T_{0} \exp \left[-\frac{\left(y-L_{t}^{\prime}\right)^{2}}{2 w_{y}^{2}}\right] \exp \left[i\left(k_{y 0}+\frac{L_{t}^{\prime \prime}}{w_{y}^{2}}\right) y\right]
$$

It is clear that the lateral shift $L_{t}^{\prime}=-d \phi / d k_{y 0}$ is the same as the one obtained by the stationary phase method [3] , and is given by

$$
\begin{aligned}
s_{t}^{p} & =\frac{s_{c}}{2 f_{0}^{2}}\left[\left(\frac{\kappa_{0}}{k_{x 0}}-\frac{k_{x 0}}{\kappa_{0}}\right)\right. \\
& \left.+\left(\frac{k_{x 0}}{\kappa_{0}}+\frac{\kappa_{0}}{k_{x 0}}\right)\left(1+\frac{\kappa_{0}^{2}}{k_{x 0}^{2}}\right) \frac{\sinh 2 \kappa_{0} a}{2 \kappa_{0} a}\right],
\end{aligned}
$$

where $s_{c}=a k_{y 0} / \kappa_{0}$. When the width of the air gap is large enough, that is, $a \gg 1 / \kappa$, the $\mathrm{GH}$ shift will tends to a constant,

$$
s_{\text {asymp }}^{p} \equiv \lim _{\kappa_{0} a \rightarrow \infty} s_{t}^{p}=\frac{2 k_{y 0}}{\kappa_{0} k_{x 0}} .
$$

With increasing the air gap the GH shift reaches a asymptotic constant, which is in agreement with the experimental results [19], and is also closely related to the counterintuitive "Hartman effect" of the tunneling delay time in the limit of an opaque barrier [23, 24].

More interestingly, what we emphasized here is that the GH shift approaches the saturation value in two different ways depending on the angle of incidence. The GH shift (9) can be expressed by the following form:

$$
\begin{aligned}
s_{t}^{p}= & \frac{g_{0}}{1+g_{0}^{2}}\left[\left(1+\frac{\kappa_{0}^{2}}{k_{x 0}^{2}}\right)\left(1+\frac{k_{x 0}^{2}}{\kappa_{0}^{2}}\right) \frac{k_{y 0}}{k_{x 0}^{2}-\kappa_{0}^{2}}\right. \\
& \left.-\frac{2 a k_{y 0}}{\kappa_{0} \sinh 2 \kappa_{0} a}\right]
\end{aligned}
$$

where $g_{0}=\left(k_{x 0}^{2}-\kappa_{0}^{2}\right) \tanh \kappa_{0} a / 2 k_{x 0} \kappa_{0}$. Keeping the next to leading term for large $a$ shows the approach to asymptotic value by

$$
s_{t}^{p} \simeq s_{a s y m p}^{p}+8 a\left(\frac{k_{y 0}}{k_{x 0}}\right)\left[\frac{k_{x 0}^{2}\left(\kappa_{0}^{2}-k_{x 0}^{2}\right)}{\left(\kappa_{0}^{2}+k_{x 0}^{2}\right)^{2}}\right] e^{-2 \kappa_{0} a} .
$$

It is clearly evident from the above expression that for $\kappa_{0}^{2}-k_{x 0}^{2}<0$ the $\mathrm{GH}$ shift increases monotonically to reach the saturation value, while for $\kappa_{0}^{2}-k_{x 0}^{2}>0$ it reaches the saturation value from above, that is, there is a hump before it attains saturation. Therefore, when the necessary condition for incident angle,

$$
\theta_{0}>\theta_{p} \equiv \sin ^{-1} \sqrt{\frac{1+n^{2}}{2 n^{2}}}
$$

is satisfied, the GH shift can approach the saturation 


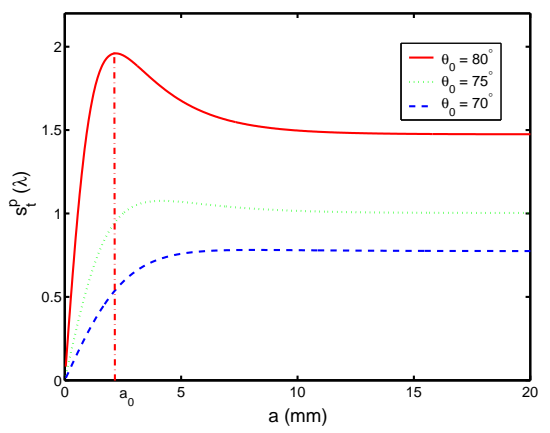

FIG. 2: (Color online) Dependence of the GH shifts (in unit of $\lambda$ ) on the air gap width $a$ in FTIR, where $\lambda=32.8 \mathrm{~mm}$ and $n=1.605$, the solid, dotted and dashed curves correspond to the incidence angles $80^{\circ}, 75^{\circ}$, and $70^{\circ}$, respectively.

limit with negative slope and can be larger than the saturation value for the intermediate values of the air gap. The position of the maximum of the hump $\left(a_{0}\right)$ is given by

$$
a_{0}=\frac{1}{2 \kappa_{0}} \frac{\kappa_{0}^{4}+3 k_{x 0}^{2} \kappa_{0}^{2}}{\left(k_{x 0}^{2}+\kappa_{0}^{2}\right)^{2}} .
$$

Fig. 2 shows that for the large $a$ the GH shift $s_{t}^{p}$ is independent of the width $a$ of the air gap hence it saturates a asymptotic constant, where $\lambda=32.8 \mathrm{~mm}$ and $n=1.605$ (corresponding to critical angle $\theta_{c}=38.5^{\circ}$ for total reflection and $\theta_{p}=56.4^{\circ}$ ) [19]. Furthermore, the $\mathrm{GH}$ shift approaches the asymptotic limit from above for $\theta_{0}>\theta_{p}$ and from below for $\theta_{0}<\theta_{p}$. These phenomenon is not due to the interference time [27], and does result from the interference between the incident and reflected beams. Of course, it can also been seen from the relationship between the GH shift and group delay discussed in Ref. 28] that the delay time in FTIR also saturates to a constant from above for $\theta_{0}>\theta_{p}$ [29] in the same way as that in the quantum tunneling for $E<V_{0} / 2$ [30, 31], since the self-interference delay time that comes from the overlap of incident and reflected waves in front of barrier is of great importance 32.

Next, what as follows we will show the influence of the beam waist width on the GH shift in FTIR. To this end, we consider the exponent of the transmission coefficient is approximated to the second-order term,

$$
\begin{aligned}
T\left(k_{y}\right) & \approx T_{0} \exp \left[\frac{1}{T_{0}} \frac{d T}{d k_{y 0}}\left(k_{y}-k_{y 0}\right)\right. \\
& \left.+\frac{1}{2} \frac{d}{d k_{y 0}}\left(\frac{1}{T} \frac{d T}{d k_{y}}\right)\left(k_{y}-k_{y 0}\right)^{2}\right]
\end{aligned}
$$

Introducing two new real parameters $F_{t}^{\prime}$ and $F_{t}^{\prime \prime}$ defined as

$$
F_{t}=F_{t}^{\prime}+i F_{t}^{\prime \prime}=-i \frac{d}{d k_{y 0}}\left(\frac{1}{T} \frac{d T}{d k_{y}}\right)
$$
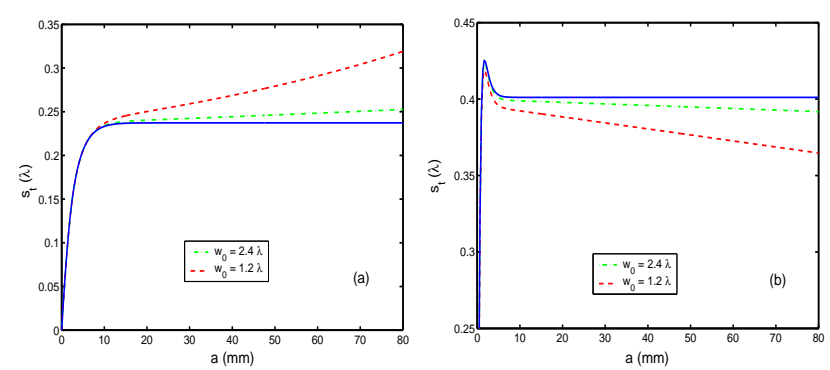

FIG. 3: (Color online) Dependence of GH shift (in unit of $\lambda$ ) on width of air gap with different beam widths, where (a) $\theta_{0}=45^{\circ}$ (b) $\theta_{0}=75^{\circ}$, and other parameters are the same as in Fig. 2. The solid corresponds to GH shift in the first-order approximation, the dashed and dotted curves correspond to the GH shifts in the second-order approximation.

then, with the phase and magnitude of $T\left(k_{y}\right)$. we have

$$
F_{t}^{\prime}=\frac{d^{2} \phi}{d k_{y 0}^{2}}
$$

and

$$
F_{t}^{\prime \prime}=-\frac{d^{2}}{d k_{y 0}^{2}} \ln |T|
$$

Substituting expression (15) into Eq. (3) using paraxial condition (7), and neglecting some unimportant factors, we finally obtain the following field of the transmitted beam at $x=a$,

$$
\begin{aligned}
\Psi_{t}(a, y) & \approx T_{0} \exp \left[-\frac{1}{2 w_{t f}^{2}}\left(y-L_{t}^{\prime}+\frac{\eta_{t} F_{t}^{\prime}}{w_{t y}}\right)^{2}\right] \\
& \times \exp \left[i\left(k_{y 0}+\frac{\eta_{t}}{w_{t y}}\right) y\right]
\end{aligned}
$$

where $\eta_{t}=L_{t}^{\prime \prime} / w_{t y}, w_{t f}=\left(w_{t y}^{2}-i F_{t}^{\prime}\right)^{1 / 2}$, and $w_{t y}^{2}=$ $w_{y}^{2}+F_{t}^{\prime \prime}$ correspond to the angular deflection, focal shift and waist-width modification, respectively [13]. The GH shift in this case can be expressed by,

$$
s_{t}=L_{t}^{\prime}-\frac{\eta_{t} F_{t}^{\prime}}{w_{t y}} .
$$

Obviously, the second term on the right-handed side of Eq. (18) is a second-order correction, which leads to the dependence of the GH shift on the beam width. In addition, it also results in the dependence of the $\mathrm{GH}$ shift on the width of air gap in the opaque barrier limit.

Fig. 3 demonstrates that the GH shift in the secondorder approximation depends on the beam width, where (a) $\theta_{0}=45^{\circ}$ (b) $\theta_{0}=75^{\circ}$, and other parameters are the same as in Fig. 2, Compared with Fig. 2 discussed above, the GH shift becomes dependent on the width $a$ in the limit of an opaque barrier, due to the second correction. When the beam width is large, that is, the divergence angle becomes small, the correction to $\mathrm{GH}$ shift 
can be neglected, thus for a well-collimated beam the $\mathrm{GH}$ shift is in agreement with that given by the stationary phase method. More importantly, Fig. 3 shows that the GH shift increases with decreasing the beam width at $\theta_{0}=45^{\circ}$, while the GH shift for $\theta_{0}=75^{\circ}$ shows a strong decrease with decreasing the beam width. As shown in Fig. 3 (a), the GH shift becomes dependent linearly on the width of air gap, because the Fourier components of the incident beam above the critical angle are strongly depressed so that the plane wave components just below the critical angle start to dominate. That is to say, when the incidence angle is larger than but close to the critical angle, the wave vector filter is more pronounced for a larger beam width, the transmission is essentially not tunneling at all, thus the GH shift increases with increasing the width $a$, as one expects classically. This also implies the violation of "Hartman effect" for the quantum tunneling in time domain [33].

Finally, we have a brief look at the microwave experiment on GH shifts. It was once argued [19] that the influences of beam width and incidence angle challenge the current descriptions of the GH shift in FTIR. Fig. 3 (a) shows the GH shift increases with deceasing beam dimension corresponding to the beam waist width, which is agreement with the experimental results in Ref. [19] where the physical parameters are the same as those in Fig. 2. In addition, it is also predicted in Fig. 3 (b) that the GH shift will decreases with deceasing beam waist, when the incidence angle is away from the critical an- gle. In a word, the improved formula of $\mathrm{GH}$ shift (18) with the modification of beam width can give better understanding of the GH shift in FTIR theoretically and experimentally.

To summary, we have investigated the GH shifts in FTIR by wave packet propagation. It is found that the GH shift in the first-order approximation of the transmission coefficient, which is exactly the expression of the $\mathrm{GH}$ shift obtained by stationary phase method, approaches the saturation value in two different ways depending on the angle of incidence. The explicit expression of the $\mathrm{GH}$ shift in the second-order approximation shows the strong dependence on the beam width. It is further shown that the GH shift with the second-order correction increases with decreasing the beam width at the small incident angles, while for the large incident angles the GH shift reveals a decrease with decreasing the beam width. All these theoretical results can be applicable to explain the experiment on GH shifts [19] and offer a hint to the better understanding of tunneling delay time in FTIR [28].

This work was supported in part by the National Natural Science Foundation of China (60806041, 60877055), the Shanghai Rising-Star Program (08QA14030), the Science and Technology Commission of Shanghai Municipal (08JC14097), the Shanghai Educational Development Foundation (2007CG52), and the Shanghai Leading Academic Discipline Program (S30105). X. Chen is also supported by Programme Juan de la Cierva of Spanish Ministry of Science and Innovation.
[1] F. Goos and H. Hänchen, Ann. Phys. 1, 333 (1947); 5, 251 (1949).

[2] H. K. V. Lotsch, Optik (Stuttgart) 32, 116 (1970); 32, 189 (1970); 32, 299 (1971); 32, 553 (1971).

[3] K. V. Artmann, Ann. Phys. (Leipzig) 2, 87 (1948).

[4] R. H. Renard, J. Opt. Soc. Am. 54, 1190 (1964).

[5] T. Sakata, H. Togo, and F. Shimokawa, Appl. Phys. Lett. 76, 2814 (2004).

[6] X. B. Yin, L. Hesselink, Appl. Phys. Lett. 89, 261108, (2006).

[7] T.-Y. Yu, H.-G. Li, Z.-Q, Cao, Y. Wang, Q.-S. Shen, Y. He, Opt. Lett. 33, 1001 (2008).

[8] C. W. Hsue and T. Tamir, J. Opt. Soc. Am. A. 2, 978 (1985).

[9] R. P. Riesz and R. Simon, J. Opt. Soc. Am. A. 2, 1809 (1985).

[10] T. Tamir, J. Opt. Soc. A. 3, 558 (1986).

[11] C.-F. Li, Phys. Rev. Lett. 91, 133903 (2003).

[12] D. Müller, D. Tharanga, A. A. Stahlhofen, and G. Nimtz, Europhys. Lett. 73, 526 (2006).

[13] C.-F. Li, Y. Zhang, X. Chen, and Q.-B. Zhu, Chin. Phys. B, 17, 1758 (2008).

[14] X. Yin, L. Hesselink, Z. Liu, N. Fang, and X. Zhang, Appl. Phys. Lett. 85, 372 (2004).

[15] F. Pillon, H. Gilles, S. Girard, M. Laroche, R. Kaiser, and A. Gazibegovic, J. Opt. Soc. Am. B 22, 1290 (2005).

[16] J. J. Cowan and B. Anicin, J. Opt. Soc. Am. 67, 1307 (1977).
[17] J. L. Agudin and A. M. Platzeck, J. Opt. (Paris) 9, 187 (1987).

[18] A. K. Ghatak, M. R. Shenoy, I. C. Goyal, and K. Thyagarajan, Opt. Commun. 56, 313 (1986).

[19] A. Haibel, G. Nimtz, and A. A. Stahlhofen, Phys. Rev. E 63, 047601 (2001).

[20] J. Broe and O. Keller, J. Opt. Soc. Am. A 19, 1212 (2002).

[21] A. M. Steinberg and R. Y. Chiao, Phys. Rev. A 49, 3283 (1994).

[22] A. A. Stahlhofen, Phys. Rev. A 62, 012112 (2000).

[23] Ph. Balcou and L. Dutriaux. Phys. Rev. Lett. 78, 851 (1997).

[24] A. Haibel, G. Nimtz, Ann. Phys. (Leipzig) 10, 707 (2001).

[25] I. R. Hooper, T. W. Preist, and J. R. Sambles, Phys. Rev. Lett. 97, 053902 (2006).

[26] T. E. Hartman, J. Appl. Phys. 33, 3427 (1962).

[27] J. C. Martinez and E. Polatdemir, Phys. Lett. A 351, 31 (2006).

[28] H. G. Winful and C. Zhang, Phys. Rev. A 79, 023826 (2009).

[29] The neccessay condition can also be derived from $\tau_{i}>\tau_{d}$ given in Ref. [28] in the limit of thin barrier.

[30] A. Paul, A. Saha, S. Bandopadhyay, and B. Dutta-Roy. Eur. Phys. J. D 42, 495 (2007).

[31] D. R. Solli, J. J. Morehead, C. F. McCormick, and J. M. Hickmann, J. Opt. A: Pure Appl. 10, 075204 (2008). 
[32] H. G. Winful, Phys. Rev. Lett. 91, 260401 (2003).

[33] V. Delgado and J. G. Muga, Ann. Phys. (New York) 248

122 (1996). 